\title{
Spermatic Cord Hemorrhage, CTCAE
}

National Cancer Institute

\section{Source}

National Cancer Institute. Spermatic Cord Hemorrhage, CT CAE. NCI Thesaurus. Code C56563.

A disorder characterized by bleeding from the spermatic cord. 